Series A

I. MATHEMATICA

481

\title{
ON GREEN'S INEQUALITIES FOR THE THIRD COEFFICIENT OF BOUNDED UNIVALENT FUNCTIONS
}

BY

OLLI TAMMI

H E L S I K I 1970

S U O M A L A INEN T I E D A K A T EM I A

doi:10.5186/aasfm.1971.481 
Communicated 8 May 1970 by LaurI Myrberg 


\section{Choosing $g$ and $D$.}

The class of bounded univalent functions is defined as follows. It consists of functions $f(z)$ analytic and univalent in the disc $U:|z|<1$ and normalized so that

$$
\left\{\begin{array}{l}
f(z)=\sum_{1}^{\infty} b_{v} z^{\nu},|f(z)|<1, \\
b_{1}=\text { a positive constant } \Rightarrow b_{1} \in(0,1] .
\end{array}\right.
$$

The corresponding class is denoted by $S\left(b_{1}\right)$. Observe that according to Schwarz's lemma $S(1)$ consists only of the identity mapping $z$.

To each $f$ there belongs the bounded function

$$
\left\{\begin{array}{l}
b_{1}^{-1} f(z)=\sum_{1}^{\infty} a_{v} z^{v}, a_{v}=\frac{b_{v}}{b_{1}}, \\
\left|b_{1}^{-1} f(z)\right|<b_{1}^{-1} .
\end{array}\right.
$$

We are going to consider coefficient inequalities for the $a_{n}$-coefficients by a method starting from an inequality due to Green's formula (cf. (2) and (3) in [8])

$$
0 \leqq \int_{D} \int_{D}\left|g^{\prime}(W)\right|^{2} d \sigma=\frac{1}{i} \int_{\partial \boldsymbol{D}} \operatorname{Re}\{g(W)\} g^{\prime}(W) d W .
$$

Here, $D$ is supposed to be a simply connected simple domain in the $W$-plane. The generating function $g$ is to be chosen so that $g^{\prime}$ is analytic and Re $g$ harmonic, in $D$ closure, $\bar{D}$.

In [5] was derived an inequality generalizing the Nehari inequality for the $S\left(b_{1}\right)$-functions. The result was sufficiently strong to maximize the coefficient $a_{3}$ for the interval $e^{-1} \leqq b_{1}<1$ of the parameter $b_{1}$. In [8], the reason for this success was made clear, in checking of the totality of corresponding extremal functions. In the generalized Nehari inequality, the generating function was in accordance with the extremal function, if $b_{1} \in\left[e^{-1}, 1\right)$. It is the most natural problem to try to find such a generating function $g$ which extends a sharp inequality for the remaining interval $0<b_{1}<e^{-1}$. 
In [1], De Temple extends the generalized Nehari inequality by applying variational technique. His result is sufficiently strong to solve the $a_{3}$-problem mentioned. In the present paper, only Green's inequality will be utilized to trying to find the corresponding extension. There appears that these two methods mentioned are able to give at least some results equivalent to each other, save the form.

For finding the generating function $g$ correctly we will make use of the information for the extremum function got by aid of Löwner's method, or by using Schiffer's differential equation. On the basis of the results obtained it is evident that when further inequalities are constructed for the $a_{n}$-coefficients, it must be most useful repeateadly to follow the guidance, obtainable in particular from Schiffer's differential equation.

Consider the function $f$ maximazing the coefficient $a_{3}>0$ for $b_{1} \epsilon$ $\left(0, e^{-1}\right)$. We get an equation for $f$ by aid of Löwner's method ([6] and [7]) as well as by means of Schiffer's differential equation (in [4], the corresponding problem for $a_{4}$ is delt with). The condition in question can be written by aid of the following sequence of equations

(4)

$$
\left\{\begin{array}{l}
\log \frac{w-1}{w+1}-\frac{2 w}{1-w^{2}}=\log z-\frac{1}{b_{1}(1+t)}\left(z-z^{-1}\right), \\
w=\sqrt{\frac{y+1}{y-t},} \\
y=\frac{1}{2}\left(f+f^{-1}\right), \\
t=\frac{1}{2}\left(d+d^{-1}\right), \text { where } d>0 \text { is the branch point of the } \\
\quad \text { forked slit of } f(U) .
\end{array}\right.
$$

Further, we introduce the connection

$$
W=\frac{w-1}{w+1}
$$

and observe that the left side of the first equation (4) can be written

$$
g(W)=x_{0} \log W+x_{1}\left(W-W^{-1}\right)
$$

with $x_{0}=1, x_{1}=-\frac{1}{2}$. Therefore, it is the most natural try to choose this $g(W)$ as a generating function in Green's inequality. Here $x_{0}$ is kept real, $x_{1}$ is complex and $d$ is an omitted ralue of $f$. By aid of the connections

$$
\begin{aligned}
& y=\frac{1}{2}\left(f+f^{-1}\right), \\
& w=\sqrt{\frac{y+1}{y-t}},
\end{aligned}
$$


(8)

$$
W=\frac{w-1}{w+1},
$$

we have to determine a proper domain $D$.

All the mappings in question are simple. They are illustrated in Figure 1, in the typical general case where $f\left(\partial K_{r}\right), K_{r} \in U$, cuts the real axes of the $f$-plane four times.

In the mapping (7), one must take care that the plane $\frac{y+1}{y-t}$ is cut along a slit, resulting

$$
w=\sqrt{\frac{y+1}{y-t}}=w(z), z \in U,
$$

to be analytic. The nature of this mapping implies that for $z_{1}, z_{2} \in U$ :

$$
z_{1} \neq z_{2} \Rightarrow w\left(z_{1}\right) \neq w\left(z_{2}\right) \text { and } w\left(z_{1}\right) \neq-w\left(z_{2}\right) \text {. }
$$

This further implies for $W=W(z)$ that

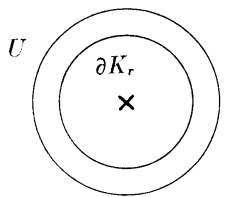

$z$
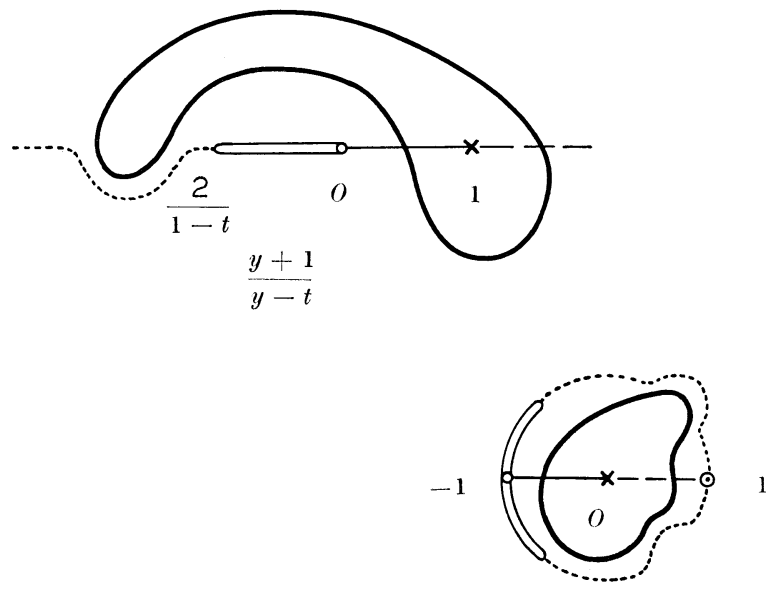

$$
W=\frac{w-1}{w+1}
$$

Fig. 1.
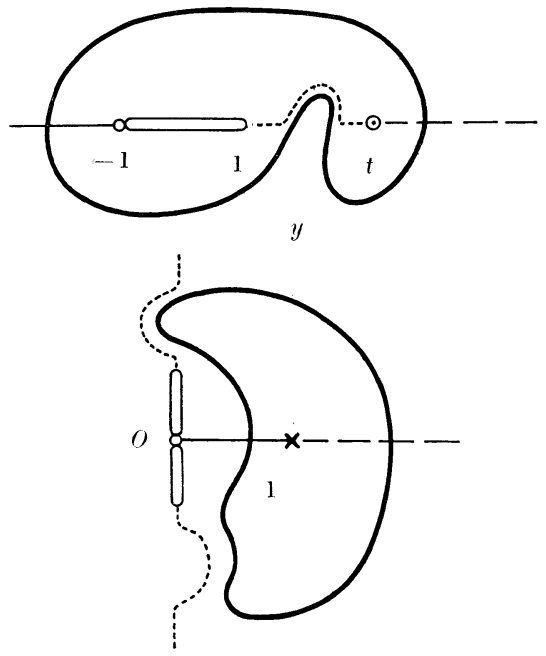

$$
W=\sqrt{\frac{y+1}{y-t}}
$$




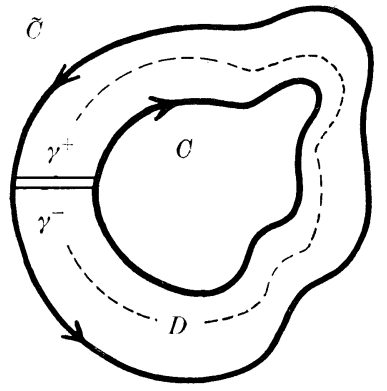

Fig. 2.

$$
z_{1} \neq z_{2} \Rightarrow W\left(z_{1}\right) \neq W\left(z_{2}\right) \text { and } W\left(z_{1}\right) \neq \frac{1}{W\left(z_{2}\right)} .
$$

Thus, we observe that $W(z)$ is a Bieberbach - Eilenberg function [2].

The curve $W\left(\partial K_{r}\right)=C$ is a simple analytic curve. Apply to $C$ the inversion $\frac{1}{W}$ to get $\tilde{C}$. According to (10) $C$ and $\tilde{C}$ have no common points. The simple ring domain determined by $-C$ and $\tilde{C}$ is cut simply connected by a properly chosen slit $\left(\gamma^{+}, \gamma^{-}\right)$. The domain $D$ thus determined has the boundary

$$
\partial D=\tilde{C} \cup \gamma^{+} \cup-C \cup \gamma^{-} .
$$

- Here we assume $C$ to be positively orientated. Hence, the Green's inequality (3) assumes the form

$$
0 \leqq \frac{1}{i} \int_{\widetilde{C}} \operatorname{Re}\{g(Z)\} g^{\prime}(Z) d Z-\frac{1}{i} \int_{C} \operatorname{Re}\{g(W)\} g^{\prime}(W) d W .
$$

Apply the inversion $W=\frac{1}{Z}$ to the first integral

$$
\begin{aligned}
& \int_{\widetilde{c}} \operatorname{Re}\{g(Z)\} g^{\prime}(Z) d Z=\int_{\widetilde{c}} \operatorname{Re}\{g(Z)\}\left[\frac{x_{0}}{Z}+x_{1}\left(1+Z^{-2}\right)\right] d Z \\
& =\int_{-C}-\operatorname{Re}\{g(W)\}\left[x_{0} W+x_{1}\left(1+W^{2}\right)\right] \frac{-d W}{W^{2}} \\
& =-\int_{C} \operatorname{Re}\{g(W)\} g^{\prime}(W) d W .
\end{aligned}
$$

Thus, (11) is reduced to the form

$$
0 \geqq \frac{2}{i} \int_{C} \operatorname{Re}\{g(W)\} g^{\prime}(W) d W .
$$




\section{An application to coefficient $a_{3}$.}

Consider the function $f(z)$ normalized by rotation so that $a_{3} \geqq 0$. Suppose that

$$
f(z) \neq d=\text { complex. }
$$

Clearly, there exist $S\left(b_{1}\right)$-functions for which the omitted values $d$ are not real, when some normalization, e.g. $a_{3} \geqq 0$, is fixed.

Now, replace $f(z)$ by the rotated function $\tau^{-1} f(\tau z),|\tau|=1$, which turns the image domain round the origin by the angle $-\arg \tau$. Choose $\tau$ so that

$$
\arg \tau=\arg d ; d=|d| \tau .
$$

Hence, $\quad \tau^{-1} f(\tau z) \neq|d|>0$. Apply the inequality (12) to the function $\tau^{-1} f(\tau z)$.

We will utilize the following development, valied in $U$ :

$$
\left\{\begin{array}{l}
w=\sqrt{\frac{y+1}{y-t}}=1+\sum_{1}^{\infty} c_{\nu} z^{\nu}, t=\frac{1}{2}\left(|d|+|d|^{-1}\right) ; \\
c_{1}=(1+t) b_{1}, \\
c_{2}=c_{1}\left(\tau a_{2}+\frac{3 t-1}{2} b_{1}\right), \\
c_{3}=c_{1}\left[\tau^{2} a_{3}+(3 t-1) b_{1} \tau a_{2}+\left(\frac{5}{2} t^{2}-t-\frac{1}{2}\right) b_{1}^{2}\right] .
\end{array}\right.
$$

Sinse $g(W(z))$ is analytic in $0<|z|<1$, the following development holds for these values of $z$ :

(16)

$$
\left\{\begin{array}{l}
g(W(z))=x_{0} \log z-\frac{2 x_{1}}{c_{1}} z^{-1}+\sum_{n=0}^{\infty} C_{n} z^{n} ; \\
C_{0}=x_{0} \log \frac{c_{1}}{2}+\frac{2 x_{1}}{c_{1}}\left(\frac{c_{2}}{c_{1}}-\frac{c_{1}}{2}\right) \\
=x_{0} \log \frac{c_{1}}{2}+\frac{2 x_{1}}{c_{1}}\left(\tau a_{2}+(t-1) b_{1}\right) \\
C_{1}=x_{1}\left[\frac{c_{1}}{2}-\frac{2}{c_{1}}\left(\frac{c_{2}^{2}}{c_{1}^{2}}-\frac{c_{3}}{c_{1}}\right)\right]+x_{0}\left(\frac{c_{2}}{c_{1}}-\frac{c_{1}}{2}\right) \\
=\frac{2 x_{1}}{c_{1}}\left[\tau^{2} a_{3}+(3 t-1) b_{1} \tau a_{2}+\left(\frac{5}{2} t^{2}-t-\frac{1}{2}\right) b_{1}^{2}\right. \\
\left.-\left(\tau a_{2}+\frac{3 t-1}{2} b_{1}\right)^{2}+\frac{c_{1}^{2}}{4}\right]+x_{0}\left(\tau a_{2}+(t-1) b_{1}\right) .
\end{array}\right.
$$


A. I. 481

The form of development (16) is exactly the same as that encountered in connection with the generalized Nehari inequality (cf. [5], (52)). Therefore the result (59) of [5] can directly be utilized to give

$$
\sum_{n=1}^{\infty} n|C|^{2} \leqq\left|\frac{2 x_{1}}{c_{1}}\right|^{2}-2 x_{0} \operatorname{Re} C_{0} .
$$

We will make use of the following consequence

$$
\left|C_{1}\right|^{2} \leqq\left|\frac{2 x_{1}}{c_{1}}\right|^{2}-2 x_{0} \operatorname{Re} C_{0}
$$

of (17). Further, $x_{0}$ will be so chosen so that $\operatorname{Re} C_{0}=0$. Hence, we have

$$
\left\{\begin{array}{l}
\left|C_{1}\right| \leqq\left|\frac{2 x_{1}}{c_{1}}\right|, \operatorname{Re} C_{0}=0 \\
\text { equality iff } C_{2}=C_{3}=\ldots=0 .
\end{array}\right.
$$

Introduce the notation

$$
a_{2}+(t-1) b_{1} \tau^{-1}=X
$$

and consider the condition

$$
\begin{gathered}
0=2 \operatorname{Re} C_{0}=2 x_{0} \log \frac{c_{1}}{2}+\frac{2}{c_{1}}\left(x_{1} \tau X+\bar{x}_{1} \tau^{-1} \bar{X}\right) ; \\
x_{0}=-\frac{x_{1} \tau X+\bar{x}_{1} \tau^{-1} \bar{X}}{c_{1}}, \\
c_{1} \log \frac{1}{2} \\
x_{0} X \tau=-\frac{x_{1} \tau^{2} X^{2}+\bar{x}_{1}|X|^{2}}{c_{1} \log \frac{c_{1}}{2}} .
\end{gathered}
$$

Observe that $\frac{c_{1}}{2}<1$, and hence formula (21) is available. This is seen by starting from the Koebe constant for the $S\left(b_{1}\right)$-functions:

$$
K\left(b_{1}\right)=\frac{b_{1}}{2-b_{1}+2 \sqrt{1-b_{1}}} \leqq|d| \leqq 1,
$$

which gives for $t=\frac{1}{2}\left(|d|+|d|^{-1}\right)$

$$
\begin{gathered}
1 \leqq t \leqq \frac{1}{2}\left[K\left(b_{1}\right)+K\left(b_{1}\right)^{-1}\right]=\frac{2}{b_{1}}-1 ; \\
b_{1} \leqq \frac{c_{1}}{2}=\frac{1+t}{2} b_{1} \leqq 1 .
\end{gathered}
$$


Equality on the right holds only for the radial slit mapping, which can be excluded.

In (21), take especially $\arg x_{1}=-\arg \tau$, which means that

$$
\bar{x}_{1}=x_{1} \tau^{2}
$$

and hence

$$
x_{0} X \tau=-\frac{2 x_{1}}{c_{1}} \frac{X^{2}+|X|^{2}}{2 \log \frac{c_{1}}{2}} \tau^{2}
$$

Taking this into consideration we obtain for $C_{1}$ :

$$
\begin{aligned}
\tau^{-2} C_{1} & =\frac{2 x_{1}}{c_{1}}\left[a_{3}+(3 t-1) b_{1} \tau^{-1} a_{2}+\left(\frac{5}{2} t^{2}-t-\frac{1}{2}\right) b_{1}^{2} \tau^{-2}\right. \\
& \left.-\left(a_{2}+\frac{3 t-1}{2} b_{1} \tau^{-1}\right)^{2}+\frac{c_{1}^{2}}{4} \tau^{-2}-\frac{X^{2}+|X|^{2}}{2 \log \frac{c_{1}}{2}}\right]
\end{aligned}
$$$$
=\frac{2 x_{1}}{c_{1}}\left[a_{3}+2(t-1) b_{1} \tau^{-1} X-\left(\frac{t^{2}}{2}-3 t+\frac{3}{2}\right) b_{1}^{2} \tau^{-2}-X^{2}-\frac{X^{2}+|X|^{2}}{2 \log \frac{c_{1}}{2}}\right]_{0} .
$$

Inequality (19) thus gives

$$
\left|[]_{0}\right| \leqq 1 \Rightarrow \operatorname{Re}[]_{0} \leqq 1,
$$

which according to (26) can be written in the form

$$
\begin{gathered}
a_{3}+2(t-1) b_{1} \operatorname{Re}\left\{\tau^{-1} X\right\}-\left(\frac{t^{2}}{2}-3 t+\frac{3}{2}\right) b_{1}^{2} \operatorname{Re}\left\{\tau^{-2}\right\} \\
-(\operatorname{Re} X)^{2}+(\operatorname{Im} X)^{2}-\frac{(\operatorname{Re} X)^{2}}{c_{1}} \leqq 1 \\
\log \frac{1}{2}
\end{gathered}
$$

Our result is thus the following inequality for $a_{3}$ :

$$
\left\{\begin{array}{l}
0 \leqq a_{3} \leqq 1+\left(\frac{t^{2}}{2}-3 t+\frac{3}{2}\right) b_{1}^{2} \operatorname{Re}\left\{\tau^{-2}\right\} \\
-2(t-1) b_{1} \operatorname{Re}\left\{\tau^{-1} X\right\}+\left(1+\frac{1}{\log \frac{c_{1}}{2}}\right)(\operatorname{Re} X)^{2} \\
\text { equality iff } \operatorname{Im} X=0 .
\end{array}\right.
$$


The right side depends on two complex numbers, $a_{2}$ and $d$, connected to $f$. It is clear that estimation of $a_{3}$ by aid of (28) can not be succesful without sufficient information of these numbers and their mutual relationships.

Finally, observe that (28) includes our former estimation. For each $S\left(b_{1}\right)$-function one is allowed to choose $d=1$. Hence, (28) yelds in this case

$$
\left\{\begin{array}{l}
a_{3}-\left(1-b_{1}^{2}\right) \leqq\left(1-\frac{1}{\log b_{1}^{-1}}\right)\left(\operatorname{Re} a_{2}\right)^{2}, \\
\text { equality iff } \operatorname{Im} a_{2}=0 .
\end{array}\right.
$$

This is the condition (79) of [5] obtained earlier from the generalized Nehari inequality.

\section{Institute of Mathematics \\ University of Helsinki}

\section{References}

[1] De Temple, D.: On coefficient inequalities for bounded univalent functions. Ann. Acad Sci. Fenn. Ser. A I, n:o 469, 1-20 (1970).

[2] Hummer, J. - Schiffer, M.: Coefficient inequalities for Bieberbach-Eilenberg functions. - Arch Rational and Mech. Anal. 32, 87-99 (1969).

[3] Nehari, Z.: Some inequalities in the theory of functions. - Trans. Amer. Math. Soc. $75,256-287$ (1953).

[4] Schiffer, M. - Tamm, O.: The fourth coefficient of bounded real univalent functions. - Ann. Acad. Sci. Fenn. Ser A I, no: 354, 1-32 (1965).

[5] -»- -»- On the coefficient problem for bounded univalent functions. Trans. Amer. Math. Soc. 140, 461-474 (1969).

[6] Tаммг, O.: On the maximalization of the coefficient $a_{3}$ of bounded schlicht functions. - Ann. Acad. Sci. Fenn. Ser A I, n:o 149, 1-14 (1953).

[7] - - On the extremal domains belonging to the coefficient $a_{3}$ of bounded schlicht functions. - Ibid. n:o 162, 1-12 (1953).

[8] -»- Grunsky type of inequalities and determination of the totality of the extremal functions. - Ibid. n:o 435, ] - 19 (1969). 